\title{
Bright Lyman-break galaxy candidates in the SDSS first data release
}

\author{
Misty C. Bentz ${ }^{1}$, Patrick S. Osmer ${ }^{1}$ and David H. Weinberg ${ }^{1}$ \\ ${ }^{1}$ Department of Astronomy, The Ohio State University, 140 W. 18th Ave, Columbus, \\ OH 43210-1173, USA
}

\begin{abstract}
We discuss the discovery of six bright Lyman break galaxy candidates in the Sloan Digital Sky Survey and the follow-up observations necessary to determine whether they are powered by star formation or by active galactic nuclei.
\end{abstract}

\section{Introduction}

The discovery of an apparently luminous $(r \approx 20.5 \mathrm{mag})$ star-forming galaxy at $z \approx 2.5$ by Bentz \& Osmer (2004) in the SDSS EDR Quasar Catalog (Schneider et al. 2002) was an unexpected surprise. Most known Lyman break galaxies (LBGs) have $R_{A B} \approx 23$ mag, which is much fainter than the limit of SDSS. A dedicated search through the SDSS DR1 produced five additional objects, as well as the original EDR object, with redshifts $2.45<z<2.80$ and $19.8<m_{r}<20.5$ (Bentz et al. 2004). These objects, by definition, have similar emission- and absorption-line properties to the LBG composite spectrum (Shapley et al. 2003, hereafter S03; see Figure 1), but there are slight differences: 1) the spectra are redder, 2) the lines are broader, and 3) the high ionization lines are stronger than the lower ionization lines.

\section{What Are These Objects?}

\subsection{Ultraluminous LBGs?}

At over 4 mag brighter than an " $L_{*}$ " LBG, these objects are expected to have star formation rates of $\sim 300-1000 \mathrm{M}_{\odot} \mathrm{yr}^{-1}$ using the continuum luminosity at $\lambda 1500 \AA$ as a proxy for star formation, without corrections for dust. The turbulence of such a system may be able to reproduce the differences observed in the spectra of the objects compared to the composite spectrum of S03. A rough estimate of the luminosity function of these objects is well above a Schecter function extrapolation of the LBG luminosity function determined by Adelberger (2000, hereafter AS00; see Figure 2). Such objects would be the most luminous members of their class and would be extremely important to understanding the formation and evolution of star-forming galaxies at high redshift. They could even be the most luminous known galaxies in the Universe.

\subsection{Gravitationally-Lensed LBGs?}

This has already been found to be the case with the galaxy MS 1512-cB58, which is an " $L_{*}$ " LBG that has been brightened by a factor of 20 by a foreground cluster (Seitz et al. 1998). The lensing hypothesis would account for the space density being higher than predicted for objects this luminous, but does not seem to be borne out by the differences in the spectra of the objects when compared with the LBG composite of S03. Also, there is no evidence in the SDSS images for lensing. 

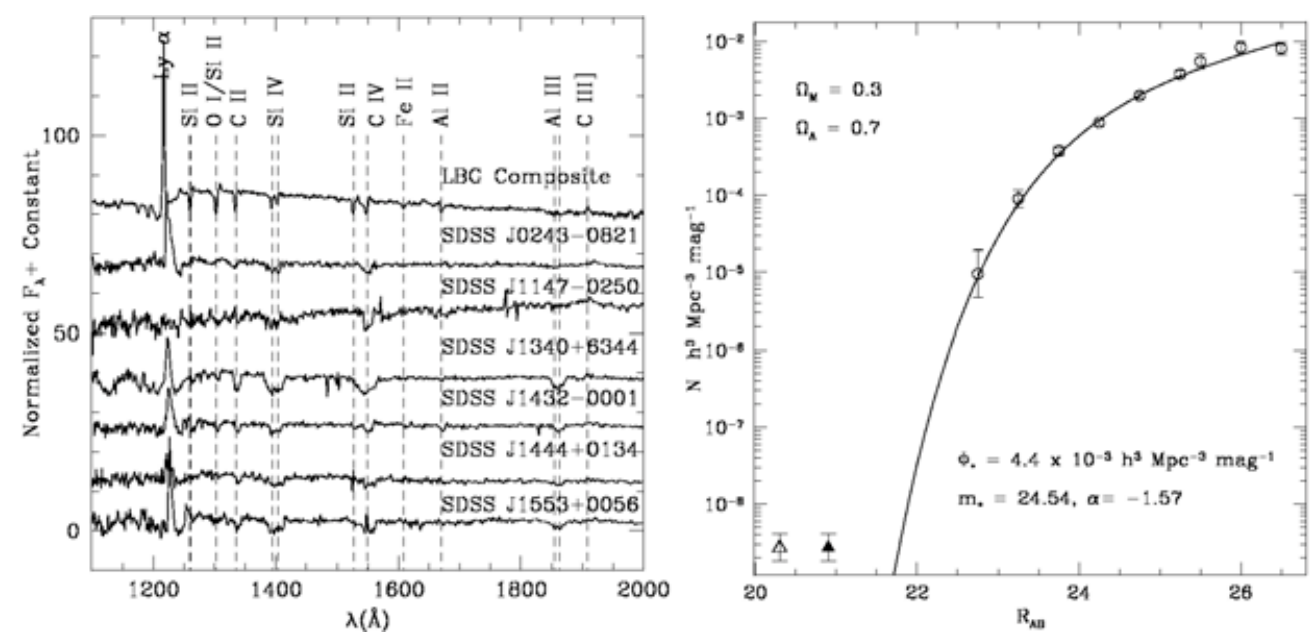

Figure 1. (left) Rest-frame spectra of the LBG composite (a; S03) and the six SDSS candidate LBGs (which have been smoothed over 5 pixels). Figure 2. (right) Luminosity function of LBGs at $z \approx 3$. Circles are data points from AS00 and the solid curve shows their Schechter function fit. The open triangle is the additional data point based on the objects described in Bentz et al. (2004), with error bars showing the Poisson noise for six objects. The filled triangle is the effect of changing from $z=2.55$, the center of the Bentz et al. (2004) redshift bin, to $z=3$, the typical redshift of the AS00 objects. Both figures are from Bentz et al. (2004)

\subsection{Unusual Quasars?}

The greater line widths in the spectra of the candidates when compared with the LBG composite spectrum of S03 could be evidence for the AGN hypothesis. The main argument against this hypothesis is the high level of similarity between the spectra of the objects and the composite spectrum. However, if they are determined to be dominated by AGN emission, these objects may represent a previously unknown class of quasar.

\section{Future Work}

Deep ACS and NICMOS imaging with HST will be used to investigate the natures and morphologies of these objects and their environments, especially as pertains to the role of gravitational lensing. In collaboration with R. Ivison and I. Smail, we will use UKIRT to obtain: 1) low-resolution HK spectroscopy to determine line intensities and ratios, 2) high-resolution long-slit $\mathrm{K}$ spectroscopy to resolve the $\mathrm{Ha} /[\mathrm{NII}]$ emission lines and search for broad AGN components, and 3) IFU spectroscopy to map the velocity structure within the object with the brightest/most extended Ha line. X-ray imaging with Chandra has also been proposed to search for hard X-ray sources within the objects and their surrounding environments as evidence of AGN activity.

\section{References}

Adelberger, K. L., \& Steidel, C. C. 2000, ApJ, 544, 218

Bentz, M. C., \& Osmer, P. S. 2004, AJ, 127, 576

Bentz, M. C., Osmer, P. S., \& Weinberg, D. H. 2004, ApJ, 600, L19

Schneider, D. P., et al. 2002, AJ, 123, 567

Seitz, S., et al. 1998, MNRAS, 298, 945

Shapley, A. E., Steidel, C. C., Pettini, M., \& Adelberger, K. L. 2003, ApJ, 588, 65 